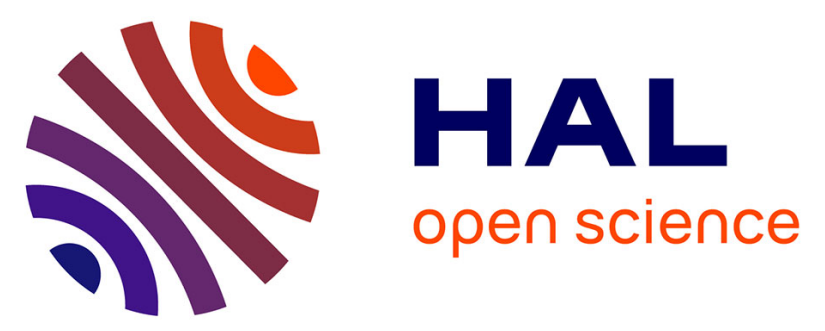

\title{
Création d'atomes d'argon excités dans des états de Rydberg en vue de l'étude de l'autodétachement électronique d'ions moléculaires SF6 -6 dans un piège quadrupolaire radiofréquence
}

\author{
G. Brincourt, M. Vedel, Y. Zerega, J. André, F. Vedel
}

\section{To cite this version:}

G. Brincourt, M. Vedel, Y. Zerega, J. André, F. Vedel. Création d'atomes d'argon excités dans des états de Rydberg en vue de l'étude de l'autodétachement électronique d'ions moléculaires SF6 -6 dans un piège quadrupolaire radiofréquence. Revue de Physique Appliquée, 1982, 17 (12), pp.813-818. 10.1051/rphysap:019820017012081300 . jpa-00245062

\section{HAL Id: jpa-00245062 https://hal.science/jpa-00245062}

Submitted on 1 Jan 1982

HAL is a multi-disciplinary open access archive for the deposit and dissemination of scientific research documents, whether they are published or not. The documents may come from teaching and research institutions in France or abroad, or from public or private research centers.
L'archive ouverte pluridisciplinaire HAL, est destinée au dépôt et à la diffusion de documents scientifiques de niveau recherche, publiés ou non, émanant des établissements d'enseignement et de recherche français ou étrangers, des laboratoires publics ou privés. 
Classification

Physics Abstracts

$07.75-35.80$

\title{
Création d'atomes d'argon excités dans des états de Rydberg en vue de l'étude de l'autodétachement électronique d'ions moléculaires $\mathrm{SF}_{6}^{-}$ dans un piège quadrupolaire radiofréquence
}

\author{
G. Brincourt, M. Vedel, Y. Zerega, J. André et F. Vedel \\ Université de Provence, Physique des Interactions Ioniques et Moléculaires (*) \\ Centre de St Jérôme, 13397 Marseille Cedex 13, France
}

(Reçu le 15 juin 1982, révisé le 14 septembre 1982, accepté le 23 septembre 1982)

\begin{abstract}
Résumé. - Nous décrivons dans ce travail les conditions expérimentales d'obtention d'atomes d'argon excités dans des états de Rydberg par bombardement électronique.

Nous précisons certaines de leurs propriétés qu'il est nécessaire de connaitre dans la perspective de l'étude de l'autodétachement électronique d'ions moléculaires $\mathrm{SF}_{6}^{-}$dans un piège quadrupolaire radiofréquence. Ces ions sont créés par interaction, à énergie quasi-nulle, de molécules $\mathrm{SF}_{6}$ avec des atomes excités dans des états de Rydberg. Dans nos conditions expérimentales, l'excitation d'atomes d'argon dans de tels états par bombardement électronique nous a conduits, en particulier, à la mise en évidence d'un angle de déflection des atomes excités, à l'évaluation de l'énergie de l'électron donnant lieu à l'attachement électronique, au nombre d'atomes excités créés, ainsi qu'à une estimation de leur durée de vie moyenne correspondante.
\end{abstract}

\begin{abstract}
In this work, we describe experimental conditions to obtain argon atoms excited in Rydberg states by an electron gun. Precisions are given about some of their properties it is necessary to know with a view to the study of electronic autodetachment of $\mathrm{SF}_{6}^{-}$molecular ions in a radiofrequency quadrupole trap. These ions are created by nearly-zero energy interaction of $\mathrm{SF}_{6}$ molecules with excited atoms in Rydberg states. In our experimental conditions, the excitation of argon atoms by electronic bombardment in such states has led us to bring to light a deflection angle of the excited atoms, an evaluation of the energy of the electrons which lead to electronic attachment, the number of created excited atoms, and an estimation of their related mean lifetime.
\end{abstract}

1. Introduction. - Le confinement d'ions au moyen de pièges quadrupolaires radio-fréquence est utilisé dans de nombreux domaines de la physique des milieux dilués, et en particulier dans celui de la physique des collisions réactives [1]. Cependant, des processus tels que l'attachement électronique, à énergie quasinulle, de molécules par des électrons thermiques semblent difficiles à mettre en ouvre car il est pratiquement impossible de réaliser une source d'électrons libres de très faible énergie dans un milieu où les champs électriques sont de l'ordre de plusieurs dizaines de volts par centimètre. La difficulté peut être contournée par l'utilisation d'atomes de Rydberg qui peuvent constituer une telle source d'électrons thermiques, méthode qui a été proposée pour réaliser l'attachement électronique de molécules $\mathrm{SF}_{6}[2,3]$.

La durée de vie de l'ion $\mathrm{SF}_{6}^{-}$ainsi obtenu, qui pourrait être supérieure à $10^{-3} \mathrm{~s}$, étant controversée, nous reprenons actuellement l'étude de cette réaction à

$\left(^{*}\right)$ Equipe de recherche associée au C.N.R.S. No 898. l'intérieur d'un piège, dispositif tout indiqué pour des mesures de durée de vie importante.

Le présent article porte sur la phase préliminaire de ce travail que constitue la création des atomes de Rydberg et la détermination de certaines de leurs propriétés qu'il est intéressant de connaître pour l'étude de la réaction.

Parmi les techniques possibles de création d'atomes excités dans des états de Rydberg, nous avons choisi celle qui consiste à exciter un jet gazeux d'argon par un canon à électrons, le jet étant orienté vers le piège où il pénètre après excitation. En effet, cette méthode d'excitation, qui est relativement simple à mettre en œuvre, permet l'accroissement des durées de vie des atomes excités, grâce à une redistribution de leur état de moment cinétique $l$ sous l'effet de leur interaction avec les électrons [4].

La non-sélectivité de cette interaction, par contre, ne permet pas de disposer d'une grande quantité d'atomes dans des états excités déterminés, contrairement aux techniques d'excitation beaucoup plus sélectives utilisant des lasers. 
2. Dispositif expérimental. -2.1 CRÉATION DES ATOMES EXCITÉs. - Dans le montage complet représenté figure 1, le jet d'argon, contenant des atomes excités dans des états de Rydberg (notés $\mathrm{Ar}^{* *}$ ), croise un jet de molécules $\mathrm{SF}_{6}$, la zone d'interaction où sont créés les ions $\mathrm{SF}_{6}^{-}$étant située au centre d'un piège quadrupolaire R.F.

L'étude préliminaire (objet de cet article) de la création et des caractéristiques des atomes excités $\mathrm{Ar}^{* *}$ a été réalisée en l'absence de jet de $\mathrm{SF}_{6}$ et en substituant au piège R.F. un dispositif de détection de ces atomes excités. Le jet d'argon est créé dans l'enceinte $\mathrm{C}_{2}$; il est obtenu en faisant communiquer un réservoir de gaz $\left(R_{1}\right)$ à la pression $\rho_{\mathrm{A}}$ avec cette enceinte, par l'intermédiaire d'une galette de microcanaux utilisée habituellement comme multiplicateur d'électrons. Ce système fonctionnant en jet multicanal (ou multijet) permet d'augmenter le débit gazeux par rapport à celui d'un canal unique, tout en conservant au jet une bonne directivité. Le jet d'argon transite ensuite dans la zone d'excitation où les atomes sont soumis à un bombardement électronique provenant d'un canon à électrons auquel est associé un système d'électrodes de commande et de focalisation (Fig. 2). L'énergie des électrons est de l'ordre de $100 \mathrm{eV}$ correspondant pratiquement à la valeur optimale de la section efficace d'excitation. Le jet pénètre alors dans l'enceinte $C_{1}$ par une petite ouverture $s_{2}$ qui détermine ses dimensions transversales. L'ouverture $s_{2}$ est suivie de plaques de déflection et de sélection $P_{1}$ et $P_{2}$, portées à des potentiels convenables, et entre lesquelles règne un champ électrique $E_{\mathrm{p}}$; elles permettent d'éliminer les particules chargées, créées par le canon, en provenance de l'enceinte $C_{2}$ (déflection) et de ne laisser passer vers le détecteur que certaines catégories d'atomes excités, les autres étant ionisés et donc défléchis par le champ $E_{\mathrm{p}}$ (sélection). Les enceintes $\mathrm{C}_{3}$ et $\mathrm{C}_{1}$ commu-

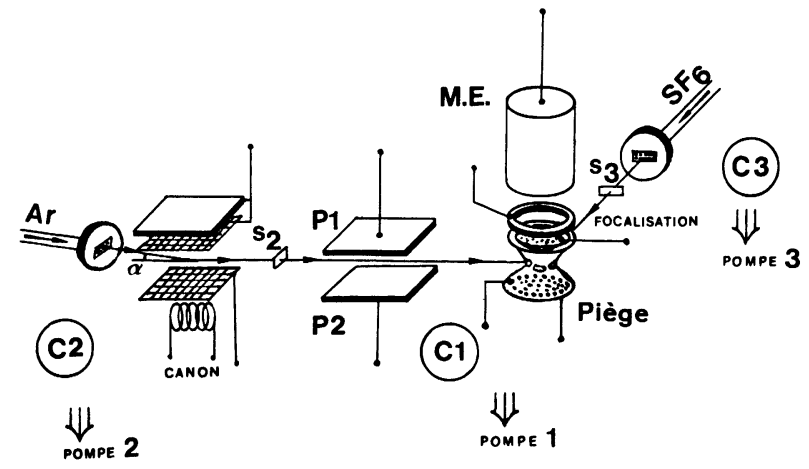

Fig. 1. - Ensemble expérimental utilisé pour la mesure de la durée de vie de l'ion $\mathrm{SF}_{6}^{-}$. L'étude expérimentale de la création des atomes excités se fait en remplaçant le piège quadrupolaire R.F. par le système de détection représenté figure 2.

[Experimental apparatus used for $\mathrm{SF}_{6}^{-}$ions radiative lifetime measure. The experimental study of the excited atoms creation is made by replacing the R.F. quadrupole trap by the detection system shown figure 2 .

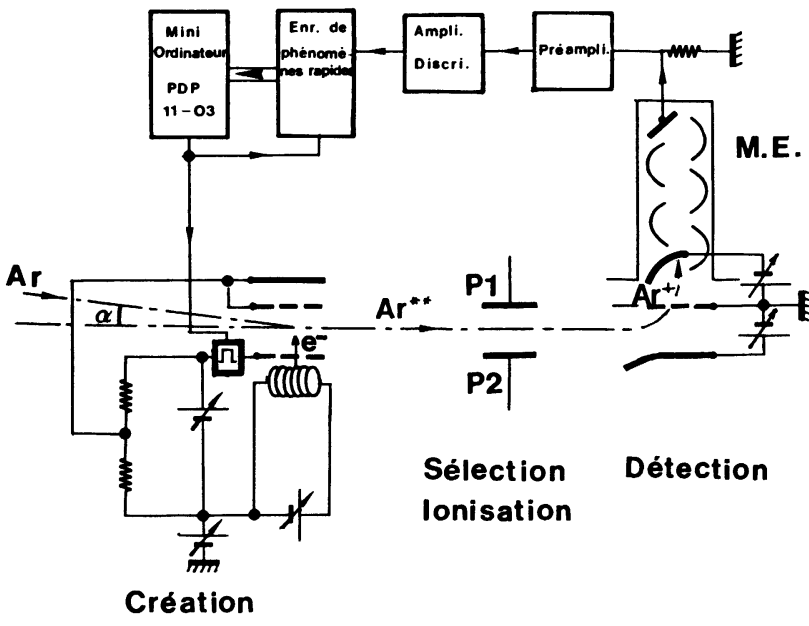

Fig. 2. - Dispositif de création (canon à électrons), de sélection (plaques $P_{1}$ et $P_{2}$ ) et de détection des atomes d'argon excités.

[Apparatus for creation (electron gun), for selection $\left(\mathbf{P}_{1}\right.$ and $P_{2}$ plates), and for detection of excited argon atoms.]

niquent par l'ouverture $s_{3}$. Chaque enceinte est reliée à un groupe de pompage de fort débit effectif (de l'ordre de $1000 \mathrm{l} / \mathrm{s}$ ) ce qui assure une pression résiduelle dans $C_{1}$ de $1 \times 10^{-7}$ torr en l'absence de jet gazeux et une pression de travail de 3 à $6 \times 10^{-7}$ torr en présence de jets.

2.2 CHOIX DE LA MÉTHODE DE DÉTECTION DES ATOMES EXCITÉs. - De nombreuses méthodes ont été proposées pour observer et caractériser des atomes excités dans des états de Rydberg. Notre dispositif utilise la technique classique d'ionisation par champ $[5,6,7]$ : la zone de détection est délimitée par un système de deux électrodes distantes de quelques millimètres entre lesquelles passe le jet d'atomes $\mathrm{Ar}^{* *}$ et où il règne un champ électrique $E_{\mathrm{d}}$, réglable, pouvant atteindre plusieurs milliers de volts par centimètre (il n'a pas été nécessaire de dépasser $4000 \mathrm{~V} / \mathrm{cm}$ ). L'électrode inférieure, portée à un potentiel positif, est constituée d'une plaque coudée à son extrémité située côté arrivée de gaz, afin que le champ d'ionisation interélectrode soit maximum dans la région par laquelle passe l'axe d'un multiplicateur d'électrons (M.E.) assurant ainsi une détection optimale. L'électrode supérieure, reliée à la masse électrique, est formée d'une grille qui est traversée par les ions $\mathrm{Ar}^{+}$; ceux-ci sont alors focalisés sur la première dynode du M.E. (voir Fig. 2).

Afin de séparer les atomes $\mathrm{Ar}^{* *}$ d'autres types de particules chargées plus rapides pouvant également produire des ions positifs, la détection est associée à une méthode de temps de vol dont le diagramme des temps (cycle) est le suivant : le canon à électrons est débloqué entre les instants $t_{0}$ et $t_{0}+\theta_{\mathrm{m}}$ avec $\theta_{\mathrm{m}} \simeq 33 \mu \mathrm{s}$; dès lors, et pendant $500 \mu \mathrm{s}$, tout événement détecté par le M.E. est préamplifié, traité dans un amplificateurdiscriminateur, puis transmis à un enregistreur de 
phénomènes rapides (Datalab) contenant 1024 canaux ouverts séquentiellement pendant $488 \mathrm{~ns}$. Le nombre d'événements par unité de temps étant faible, nous avons recours à la répétition d'un grand nombre de ces cycles pour obtenir un signal de bonne visibilité. Pour cela, le déroulement de l'expérience est piloté par un miniordinateur PDP 11/03. Il s'ajoute alors à chaque cycle un délai supplémentaire de $32 \mathrm{~ms}$ nécessaire au transfert des données de l'enregistreur vers une mémoire tampon du calculateur. Un programme séquence l'ensemble de ces opérations. La sauvegarde des enregistrements se fait sur disque souple afin de permettre un traitement ultérieur des données. La figure 3 représente, à titre d'exemple, plusieurs signaux d'ions relatifs à des nombres de cycles différents. On constate l'accroissement de la visibilité du signal avec le nombre de cycles.

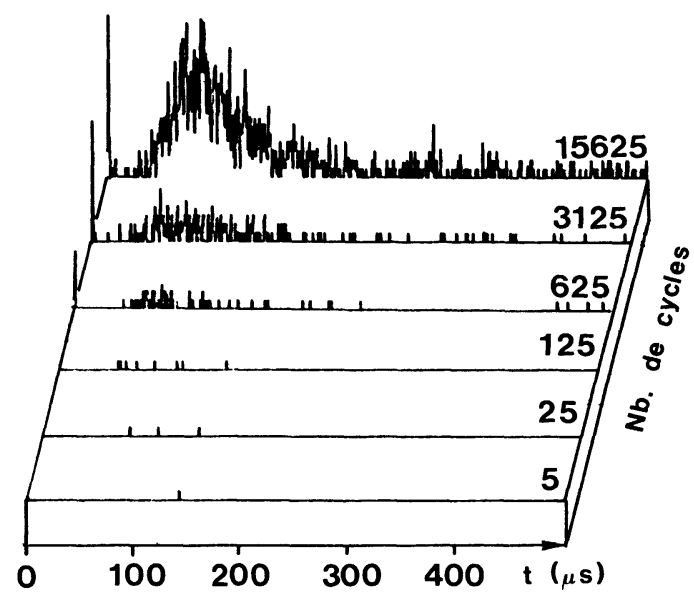

Fig. 3. - Evolution du signal observé en fonction du nombre de cycles d'enregistrement.

[Evolution of the observed signal versus the number of recording cycles.]

3. Résultats. - 3.1 Mise eN ÉVIDENCE DES ATOMES D'Ar EXCITÉs. - La figure $4 a$ montre un enregistrement typique de 20000 cycles. Afin d'en améliorer la visibilité, les 1024 canaux de comptage ont été regroupés soit par 8 (Fig. $4 b$ ) soit par 16.

Les courbes font apparaître trois zones caractéristiques. Un signal important mais bref (zone I) s'étend entre 0 et $20 \mu \mathrm{s}$; il représente des événements produits par des particules chargées rapides, créées directement ou indirectement par le canon et qui ont pu pénétrer dans l'enceinte $C_{1}$ malgré la présence des plaques de déflection $P_{1}$ et $P_{2}$. Après un temps de vol correspondant au temps de transit des atomes excités de la zone du canon à électrons au détecteur, il apparaît le signal correspondant à l'ionisation par champ de ces atomes, et qui s'étend entre $40 \mu$ s et $250 \mu$ s environ (zone II). L'existence de ce temps de vol entre les zones I et II assure une bonne séparation des signaux correspondants. La dernière zone (zone III) comprend

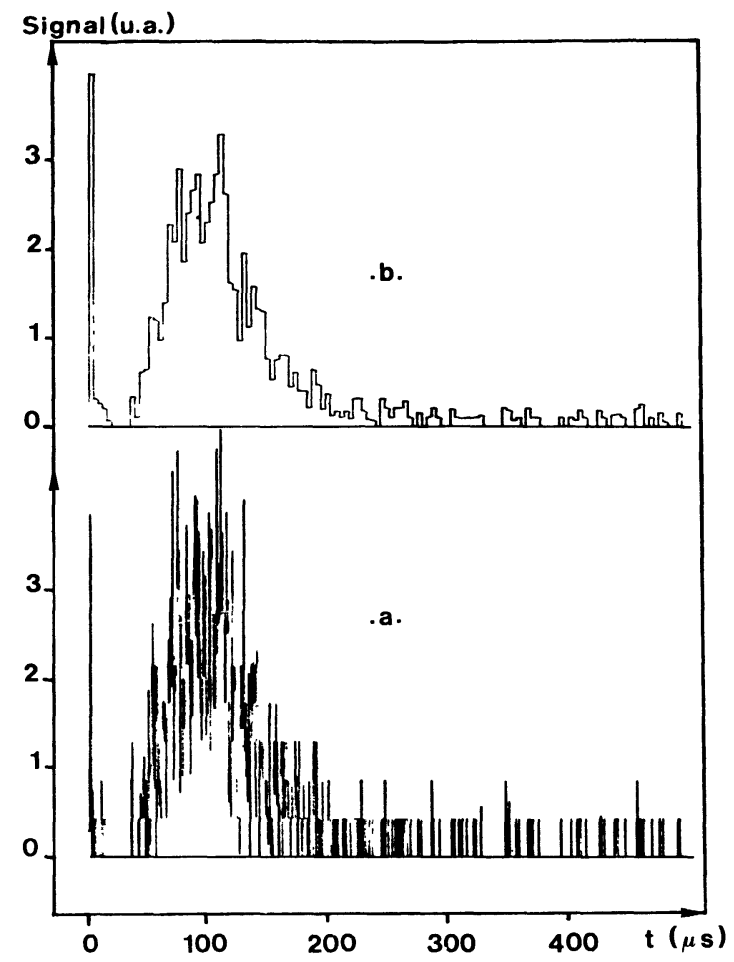

Fig. 4. - Signal correspondant à un enregistrement de 20000 cycles $(4 a)$. Le même enregistrement est représenté avec groupement par 8 des canaux de comptage (4b).

[Signal corresponding to 20000 recording cycles (4a). The same recording is given with a grouping by 8 of the counting channels $(4 b)$.]

uniquement le bruit stationnaire de l'ensemble du dispositif électronique dont l'amplitude peut être soustraite statistiquement du signal de la zone II. On obtient alors le signal " utile " de la figure $5 a$ dont l'interprétation fait l'objet du paragraphe 3.2.

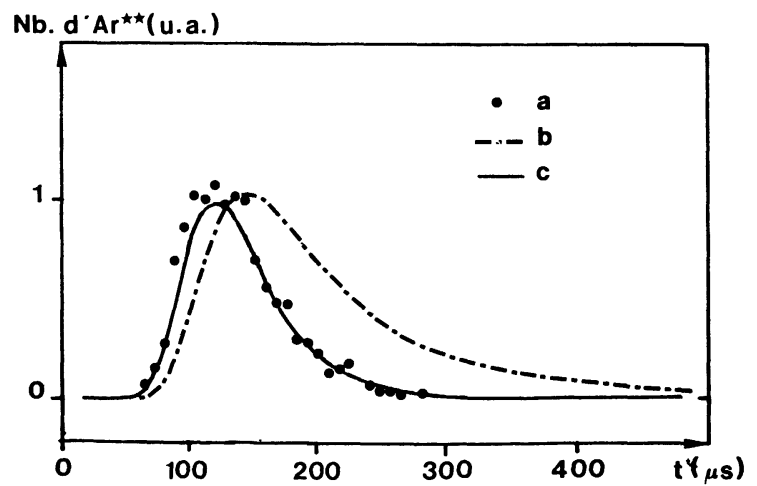

Fig. 5. - Signal expérimental (5a) auquel on a retranché le bruit ( $(\bullet)$. Courbes théoriques déduites des relations (1) et (2) : 5b : atomes stables (-- ); $5 c$ : atomes excités de durée de vie $68 \mu$ s (-).

[Experimental signal (5a) after noise substracting (๑). Theoretical curves deduced from relations (1) and (2) : $5 \mathrm{~b}$ : Stables atoms (- - ) $5 \mathrm{c}$ : Excited atoms with $68 \mu \mathrm{s}$ lifetime $(-)$.] 
Dans les conditions optimales de fonctionnement, le nombre d'événements détectés est de l'ordre de 0,08 par cycle, pour une pression d'argon en amont de la pastille de jet de $10^{-1}$ torr, une durée d'excitation électronique de $33 \mu \mathrm{s}$, et un courant électronique correspondant de l'ordre du $\mathrm{mA}$. Le faible nombre d'événements obtenus justifie donc le dispositif d'acquisition et de traitement de données que nous utilisons.

\subsection{INTERPRÉTATION DES RÉSULTATS. - Le nombre} d'événements détectés $\frac{\partial N(t)}{\partial t}$ entre les instants $t$ et $t+\mathrm{d} t$ est évidemment relié, en particulier, aux paramètres géométriques et cinétiques du canon à électrons et du jet. Pour une population d'atomes stables, la quantité $\frac{\partial N(t)}{\partial t}$ s'exprime par :

$$
\frac{\partial N(t)}{\partial t} \mathrm{~d} t=\left[C \int_{0}^{\theta_{\mathrm{m}}} \int_{\frac{D-d}{t-\theta}}^{\frac{D}{t-\theta}} v_{\mathrm{a}} \cdot f\left(v_{\mathrm{a}}\right) \cdot \mathrm{d} v_{\mathrm{a}} \cdot \mathrm{d} \theta\right] \mathrm{d} t
$$

avec $f\left(v_{\mathrm{a}}\right) \propto v_{\mathrm{a}}^{2} \exp \left(-\frac{v_{\mathrm{a}}^{2}}{2 \sigma^{2}}\right)$ et $\sigma=\sqrt{\frac{k T}{m}}$. Dans cette relation : $T$ est la température du gaz utilisé $(\sim 400 \mathrm{~K}), d$ la longueur de la zone d'interaction du gaz avec le canon à électrons, $D$ la distance séparant le canon du détecteur, $\theta_{m}$ la durée du bombardement électronique, $v_{\mathrm{a}}$ la vitesse moyenne des atomes d'argon et $C$ une constante.

La courbe $5 b$, correspondant à la relation (1), relative à des atomes stables, fait ressortir que les événements observés (Fig. 5a) sont statistiquement plus rapides, ce qui montre leur caractère métastable; en effet, un atome excité a d'autant plus de chances de parcourir une distance donnée avant de se désexciter qu'il est plus rapide, ce qui conduit à une loi de répartition des instants d'arrivée, au niveau du détecteur, différente de celle qu'on observerait dans le cas d'atomes excités de durée de vie infinie.

Si on considère un modèle de loi de durée de vie, il est possible de relier la forme du signal calculé à la valeur des paramètres de cette loi. Ainsi, pour une loi exponentielle (caractérisée par un temps de relaxation $\tau)$, l'évolution du nombre d'atomes excités dans des états de Rydberg peut s'écrire :

$$
\begin{aligned}
\frac{\partial N(t, \tau)}{\partial t} \mathrm{~d} t=\left[\rho_{0} \gamma\right. & \int_{0}^{\theta_{\mathrm{m}}} \exp \left(-\frac{t-\theta}{\tau}\right) \times \\
& \left.\left\{\int_{\frac{D-d}{t-\theta}}^{\frac{D}{t-\theta}} v_{\mathrm{a}} \cdot f\left(v_{\mathrm{a}}\right) \cdot \mathrm{d} v_{\mathrm{a}}\right\} \mathrm{d} \theta\right] \mathrm{d} t
\end{aligned}
$$

où $\rho_{0}$ est la densité du jet gazeux au niveau du canon à électrons et $\gamma$ la section efficace de création des atomes excités.

Dans le cadre de ce modèle on peut établir une relation théorique $\langle t\rangle_{\mathrm{th} .}=h(\tau)$ où $\langle t\rangle_{\mathrm{th}}$. est l'espérance mathématique de l'instant d'arrivée des atomes excités au niveau du détecteur et $h$ une fonction. Parallèlement, les courbes expérimentales fournissent une valeur $\langle t\rangle_{\text {exp. }}$. Si on pose $h\left(\tau^{*}\right)=\langle t\rangle_{\text {exp. }}, \tau^{*}$ constitue une estimation de la durée de vie moyenne des atomes excités. Par exemple, pour $E_{\mathrm{p}}=200 \mathrm{~V} / \mathrm{cm}$, l'injection de la valeur de $\tau^{*}$ correspondante dans (2) conduit à la courbe $5 c$ qui se superpose pratiquement à la courbe expérimentale $5 \mathrm{a}(\mathrm{Fig}$. 5) relative à la même valeur de $E_{\mathrm{p}}$. Pour cette comparaison, l'origine des temps de la courbe expérimentale a été ramenée à l'instant de déclenchement du canon à électrons.

Ceci montre que, malgré le caractère très simplifié du modèle exponentiel choisi, celui-ci rend néanmoins assez bien compte des propriétés temporelles observées.

La figure 6 représente plusieurs courbes expérimentales obtenues pour diverses valeurs du champ électrique $E_{\mathrm{p}}\left(E_{\mathrm{d}}=4000 \mathrm{~V} / \mathrm{cm}\right)$. Elles conduisent à des valeurs de $\tau^{*}$ qui vont de $68 \mu$ s̀ à $52 \mu$ s pour des valeurs de $E_{\mathrm{p}}$ s'échelonnant respectivement de $200 \mathrm{~V} / \mathrm{cm}$ à $8000 \mathrm{~V} / \mathrm{cm}$ (Fig. 7a). La diminution de l'aire des signaux et celle de $\tau^{*}$ lorsque $E_{\mathrm{p}}$ croît, montrent que le signal est produit, de façon caractéristique, par des atomes excités dans des états de Rydberg.

On peut songer à utiliser les valeurs de $\tau^{*}$ déterminées par la méthode précédente pour avoir un ordre de grandeur de la valeur de $n$ correspondante dans la population d'atomes excités. En effet, il existe un modèle [4] qui conduit à une durée de vie moyenne, en fonction du nombre quantique principal $n$ (corrigé du défaut quantique) pour des atomes excités d'hélium et en présence de transfert de moment cinétique orbital, donnée par :

$$
\tau(n)=3,5 \times 10^{-10} n^{4,11}(\mathrm{~s}) .
$$

Par exemple pour $\tau^{*}=60 \mu$ s qui est notre valeur expérimentale moyenne, la relation (3) donne $n \simeq 19$.

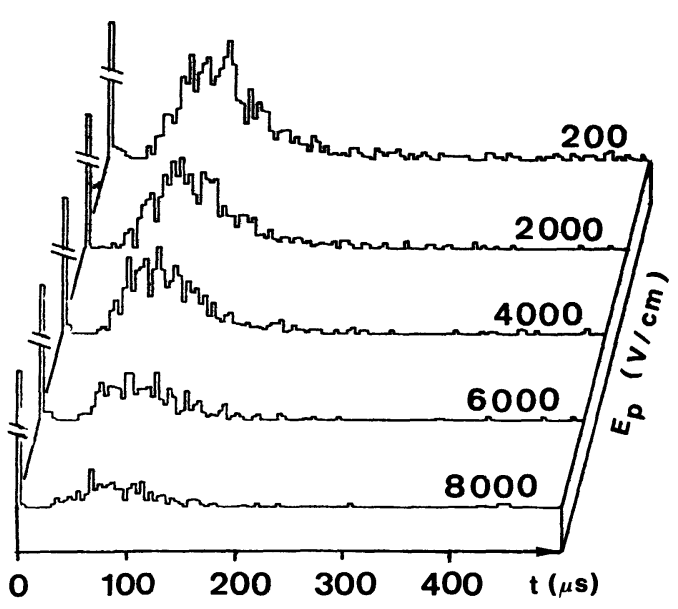

Fig. 6. - Evolution du signal observé en fonction du champ électrique $E_{\mathrm{p}}$.

[Evolution of the observed signal versus the electric field $E_{\mathbf{p}}$ ]] 


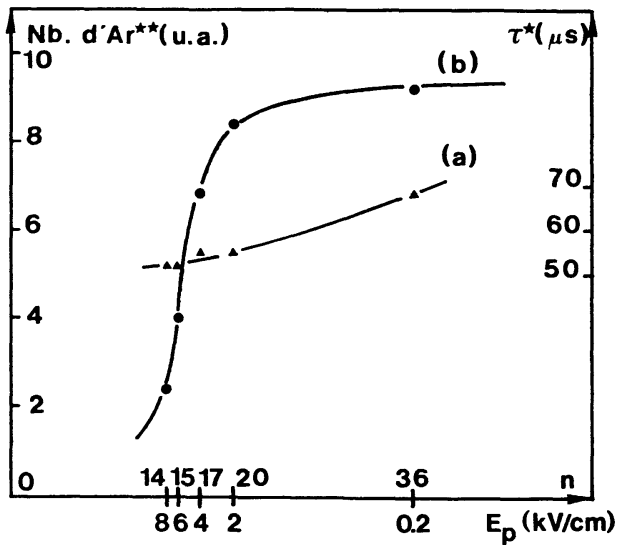

Fig. 7. $-a$ ) Evolution de $\tau^{*}$ en fonction du champ électrique $E_{\mathrm{p}} ; b$ ) Nombre relatif d'ions détectés en fonction de $E_{\mathrm{p}}$. La double échelle horizontale traduit la relation (4).

[a) Evolution of $\tau^{*}$ versus the electric field $E_{\mathrm{p}} ; b$ ) Relative number of detected ions versus $E_{\mathrm{p}}$. The double horizontal scale is related to the relation (4).]

Par ailleurs, on peut considérer la relation suivante qui lie la valeur inférieure de $n$, correspondant aux atomes qui seront ionisés par champ électrique, à la valeur $E_{\mathrm{p}}$ du champ :

$$
E_{\mathrm{p}}(\mathrm{V} / \mathrm{cm})=\frac{3,2 \times 10^{8}}{n^{4}}
$$

Cette relation est illustrée par la double échelle horizontale de la figure 7 . Sur cette figure, nous avons reporté la courbe 7b qui représente le nombre relatif $N$ d'ions détectés (échelle arbitraire) en fonction du champ $E_{\mathrm{p}}$ et pour une valeur de $E_{\mathrm{d}}$ égale à $4000 \mathrm{~V} / \mathrm{cm}$. Comme prévu, ce nombre décroît très vite lorsque $E_{\mathrm{p}}$ tend vers $E_{\mathrm{d}}$, sans toutefois atteindre zéro pour $E_{\mathrm{p}}=E_{\mathrm{d}}$. Ce désaccord peut s'expliquer par le fait que des atomes qui ont été sélectionnés en $n$ et $l$ par les plaques $P_{1}$ et $P_{2}$ peuvent être l'objet, le long du trajet qui sépare le détecteur des plaques, d'une réorganisation de ces paramètres sous l'action des collisions et/ou du rayonnement du corps noir. Par exemple, des collisions peuvent intervenir malgré une pression de travail faible $\left(\leqslant 10^{-6}\right.$ torr $)$ du fait de la section efficace très importante des atomes excités. Ces interactions peuvent alors entraîner [4] une ionisation, au niveau du détecteur, pour des valeurs de champ correspondant $E_{\mathrm{d}}$ inférieures à $E_{\mathrm{p}}$. C'est bien ce que nous observons expérimentalement (Fig. $7 b$ ).

En conclusion, et au vu de nos résultats, on peut avancer que la valeur moyenne de $n$ que nous obtenons se situe autour de 19.

3.3 EFFETS DES COLLISIONS ÉLECTRONIQUES SUR LES TRAJECTOIRES DES ATOMES EXCITÉS. - Une collision entre un électron et un atome s'accompagne d'un transfert d'impulsion qui modifie leurs trajectoires incidentes, celle de l'atome étant modifiée d'un angle $\alpha$.

En l'absence d'un modèle de diffusion, un calcul simple permet de connaître l'angle de déviation maximal $\alpha_{M}$ en fonction de l'énergie des électrons, de leur direction et de la vitesse $v_{\mathrm{a}}$ des atomes subissant l'interaction. L'angle $\alpha$ est une fonction décroissante de $v_{\mathrm{a}}$. Dans nos conditions expérimentales, si on prend pour $v_{\mathrm{a}}$ la valeur de lả vitesse moyenne des atomes dans le jet, le calcul donne $\alpha_{\mathrm{M}} \simeq 22^{\circ}$, valeur qui autorise un angle compris entre 0 et $22^{\circ}$.

La figure 8 représente, expérimentalement, le nombre d'atomes détectés en fonction de $\alpha$. Elle est obtenue par modification de l'angle d'incidence du dispositif de jet de gaz, la commande angulaire pouvant se faire de façon continue de l'extérieur de l'enceinte. Un soin particulier a été apporté à l'alignement et à l'étalonnage de ce dispositif. Cette courbe fait apparaître un angle de déviation moyen des atomes excités de l'ordre de 5 , en accord avec la limite supérieure calculée. L'écart important entre les deux valeurs s'explique partiellement par le fait que parmi les atomes excités, les plus lents d'entre eux, donc les plus déviés, se sont désexcités avant d'être détectés; ils ne contribuent donc pas à la courbe 8 .

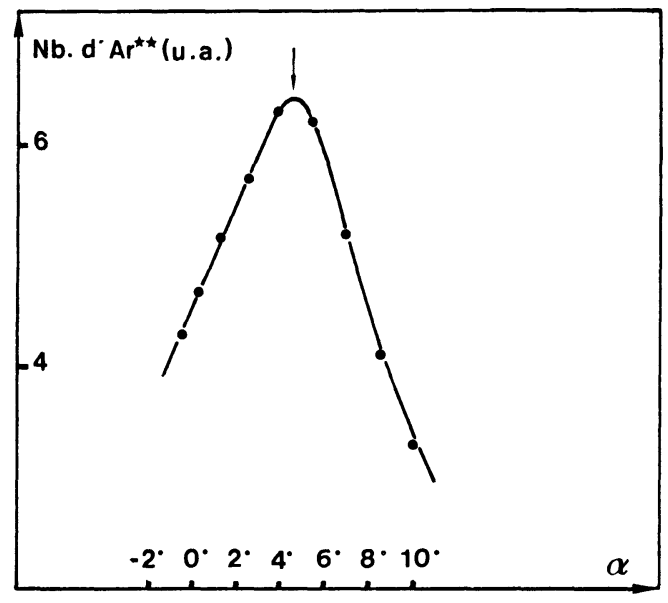

Fig. 8. - Nombre d'atomes excités détectés en fonction de l'angle $\alpha$ que fait le jet gazeux avec la direction de détection.

[Number of excited atoms detected versus the angle $\alpha$ made by the gas jet with the direction of detection.]

4. Conclusion. - L'étude effectuée a permis, dans nos conditions expérimentales, de déterminer l'ordre de grandeur du nombre d'atomes d'argon excités dans des états de Rydberg $(0,08$ par cycle et pour une durée d'excitation électronique de $33 \mu \mathrm{s}$ ) et de préciser certaines de leurs propriétés comme leur degré d'excitation, leur durée de vie et leur direction après création. La valeur moyenne trouvée pour $n(\sim 19)$ est proche de celle obtenue dans des conditions expérimentales voisines $(n \geqslant 20)$ par J. P. Astruc et al. [10].

Malgré le faible nombre d'atomes excités créés, il apparaît néanmoins possible de mettre en évidence la 
réaction :

$$
\mathrm{A}_{\mathrm{r}}^{* *}+\mathrm{SF}_{6} \rightarrow \mathrm{SF}_{6}^{-}+\mathrm{A}_{\mathrm{r}}^{+}
$$

que nous nous proposons d'étudier, en particulier grâce au dispositif d'acquisition et de traitement de données dont nous disposons. Par ailleurs, l'importance du taux de réaction associé $\left(\gtrsim 10^{-7} \mathrm{~cm}^{3} \mathrm{~s}^{-1}\right)[8,2,9]$, entraîne une probabilité de cette réaction voisine de 1 . On peut donc s'attendre à un nombre d'événements $\mathrm{SF}_{6}^{-}$détectés qui soit de l'ordre de grandeur de celui des atomes d'argon excités. Enfin, la formule de Rydberg appliquée à notre cas conduit à une énergie électronique transférée à la molécule $\mathrm{SF}_{6}$ de $\sim 30 \mathrm{meV}$ ce qui exclut toute perturbation notable de son énergie interne dans cette réaction.

Remerciements. - Les auteurs remercient messieurs J. P. Schermann et R. Barbé du Laboratoire de Physique des Lasers (Université de Paris Nord) pour leurs conseils et leur aide amicale. Cette étude a été réalisée dans le cadre de l'ATP : "Processus élémentaires et objets simples en physique atomique et moléculaire. »

\section{Bibliographie}

[1] SChUessler, H. A., Progress in atomic spectroscopy (Plenum Press, N. Y.) 1979, p. 999.

[2] Hotop, H. and Niehaus, A., J. Chem. Phys. 47 (1967) 2506.

[3] Astruc, J. P., Barbe, R., Schermann, J. P., J. Phys. B 12 (1979) 377-381.

[4] Schiavone, J. A., Donohue, D. E., Herrick, D. R. and Freund, R. S., Phys. Rev. A 16 (1977) 48.

[5] Ducas, T. W., Littman, M. G., Freeman, R. R. and KLEPPNER, D., Phys. Rev. Lett. 35 (1975) 366.
[6] Stebbings, R. F., Latimer, C. J., West, W. P., Dunning, F. B. and Cook, T. B., Phys. Rev. A 12 (1975) 1453.

[7] Van RaAn, A. F. J., Braun, G. and Raith, W., $J$. Phys. B 9 (1976) L-173.

[8] Matsuzawa, M., J. Phys. Soc. Jap. 32 (1972) 1088-94.

[9] Odom, R. W., Smith, D. L., Futrell, J. H., J. Phys. $B 8$ (1975) 1349 et références citées.

[10] Schermann, J. P., Astruc, J. P. et Barbe, R., 8e colloque sur la Physique des Collisions Atomiques et Moléculaires. Louvain la Neuve, juillet 1980. 